\title{
A MAC-Layer Differentiated Service Model in IEEE 802.11e WLANs
}

\author{
Jeng Farn Lee ${ }^{1,3}$, Wanjiun Liao ${ }^{1,2}$, and Meng Chang Chen ${ }^{3}$ \\ ${ }^{1}$ Department of Electrical Engineering, National Taiwan University, Taipei, Taiwan \\ ${ }^{2}$ Graduate Institute of Communication Engineering, National Taiwan University, Taipei, Taiwan \\ ${ }^{3}$ Institute of Information Science, Academia Sinica, Taipei, Taiwan \\ Email:wjliao@ntu.edu.tw
}

\begin{abstract}
In this paper, we provide a differentiated service model in IEEE 802.11e wireless local area networks (WLANs). In our service model, a new mechanism called Differentiated Service - EDCA (DSEDCA) is proposed to provide both strict priority and proportional fair service for IEEE 802.11 WLANs. The EDCA parameter set of lower priority traffic is carefully designed, and the backoff intervals are determined according to the distributed scheduling discipline DFS. Furthermore, a hierarchical link sharing model is proposed for IEEE 802.11e WLANs, which allocates different amount of link resource for AP and mobile stations. The performance of DS-EDCA and EDCA is compared via ns2 simulations. The results show that DS-EDCA outperforms the original EDCA in terms of the support for both strict priority and weighted fair service. Most importantly, DS-EDCA can be easily implemented, and is compatible to the IEEE 802.11 Standard.
\end{abstract}

Keywords -- EDCA, 802.11e, strict priority, weighted fair service.

\section{INTRODUCTION}

IEEE $802.11 \mathrm{e}$ [1] is a supplementary standard of 802.11 to provide QoS for different kinds of applications. In 802.11e, QoS is supported with a new access method called the Hybrid Coordination Function (HCF). HCF defines two medium access mechanisms. In this paper, we focus only on the HCF contention-based channel access mechanism, referred to as Enhanced Distributed Channel Access (EDCA).

The EDCA of 802.11e, an enhanced version of 802.11 DCF, provides priority-based service for different access categories (ACs). With EDCA, a station can implement up to four $\mathrm{ACs}$, which are $\mathrm{AC} \mathrm{VO}, \mathrm{AC} \mathrm{VI}, \mathrm{AC} \mathrm{BE}$, and $\mathrm{AC} \mathrm{BK}$, corresponding to voice, video, best effort, and background traffic, respectively. Each $\mathrm{AC}$ is associated with one backoff entity and the operation of each backoff entity is similar to that of legacy DCF. The backoff entities are prioritized according to AC-specific parameters called the EDCA parameter set composed of Arbitrary Inter-Frame Space Number (AIFSN[AC]), minimum contention window $(C W \min [\mathrm{AC}])$, and maximum contention window $(C W \max [\mathrm{AC}])$. The
AIFSN $[\mathrm{AC}]$ is used to determine the duration of Arbitrary IFS (AIFS[AC]) according to

$\operatorname{AIFS}[A C]=\operatorname{SIFS}+\operatorname{AIFSN}[A C] \times$ aslotTime, $\operatorname{AIFSN}[A C] \geq 2$, where aslotTime is the duration of a slot.

With 802.11e EDCA, only priority-based service is supported. Such priority-based service, while allowing service differentiation for flows of different priorities, cannot ensure minimum guaranteed or expected throughputs. In addition, it cannot allow service amounts in proportion to the demands of flows. This calls for strict priority and weighted fair service to be supported by EDCA.

To provide weighted fair service, fair queuing is required to be implemented in EDCA. The major challenge of fair queuing in WLANs is that service provisioning must be fully distributed. This renders existing centralized mechanisms (e.g., [5] for wired networks and [6] for wireless networks in which base stations are involved) inappropriate. Some scheduling disciplines have been proposed for weighted fairness at the link or MAC layer of legacy 802.11 DCF, including Distributed Fair Queuing (DFS) [7], Priority-based fair Medium Access Control (P-MAC) [8], and Distributed Deficit Round Robin (DDRR) [9]. The typical approach of these efforts is to tune one of the three 802.11 MAC parameters, i.e., Backoff Interval (BI), Contention Window (CW), and Inter-Frame Space (IFS), based on certain fair queuing mechanisms.

The efforts described above all aim at providing stationbased service differentiation, rather than AC-based QoS service provided by EDCA. So far, no mechanism has been designed for EDCA to provide proportional fairness service. Adaptive Fair Enhanced DCF (AFEDCF) [11] is the only mechanism which implements fairness among flows of the same priority for 802.11e EDCA. However, AFEDCF cannot provide weighted fair service among different ACs and thus is not a proportional fairness mechanism.

In this paper, we study the provision of differentiated service model for IEEE 802.11e WLANs. In this model, both strict priority and weighted fair service are provided 
by EDCA. The resource is first allocated to flows of higher priority. The remaining bandwidth is then shared proportionally among the other service class according to their assigned weights. We propose a new mechanism called Differentiated Service -- EDCA (DS-EDCA) for the proposed service model in IEEE 802.11e EDCA systems. The performance of DS-EDCA is evaluated via simulations based on ns-2. Note that the mechanism may also be used to provide strict priority or proportional fairness service only.

The rest of the paper is organized as follows. In Sec. II, the operations of DS-EDCA are described. In Sec. III, a hierarchical link sharing model in IEEE 802.11e WLANs is proposed. In Sec. IV, the performance of the proposed mechanism based on ns-2 is evaluated. Finally, the paper is concluded in Sec. V.

\section{II.DIFFERENTIATED SERVICE - ENHANCED DISTRIBUTED CHANNEL ACCESS (DS-EDCA)}

In this section, we describe the proposed mechanism called DS-EDCA for IEEE 802.11 WLANs. With DSEDCA, 802.11e EDCA is enhanced by using Distributed Fair Scheduling (DFS) in each backoff entity to provide weighted fair service for flows of different ACs. The strict priority is ensured by carefully assigning the EDCA parameter set of lower priority traffic. In what follows, we will first describe the design principle of DS-EDCA and give an overview of DFS. Then, we will present the operations of DS-EDCA based on DFS.

\section{A. Distributed Weighted Fair Service Discipline for 802.11e EDCA}

EDCA provides priority-based service for different ACs, not for different stations. The backoff entity of each AC is used to contend different ACs within the same station and the ACs in different stations. Multiple backoff entities are used within one EDCA station to improve the channel utilization. This can also reduce the delay of each AC thanks to the eliminating of the head of line problem.

\section{B. Distributed Fair Scheduling (DFS)}

DFS is designed to emulate Self-Clocked Fair Queuing (SCFQ) [5] in a distributed manner. In SCFQ, which attempts to emulate GPS, a virtual clock is maintained by the central coordinator in the system. Let $v(t)$ denote the virtual clock at real time $t, P_{i}^{k}$ be the $\mathrm{k}^{\text {th }}$ packet of flow $i$, and $A_{i}^{k}$ be the real arrival time of $P_{i}^{k}$. Let $L_{i}^{k}$ denote the packet size of $P_{i}^{k}$. A start tag $S_{i}^{k}$ and a finish tag $F_{i}^{k}$ are associated with each packet $P_{i}^{k}$, given by

$$
\begin{aligned}
& S_{i}^{k}=\max \left\{v\left(A_{i}^{k}\right), F_{i}^{k-1}\right\} \\
& F_{i}^{k}=S_{i}^{k}+\frac{L_{i}^{k}}{\phi_{i}}
\end{aligned}
$$

where $\phi_{i}$ is the assigned weight for station $i$.

The system virtual clock is set to 0 initially, and is updated to the finish tag of the packet transmitted on the output link. In SCFQ, packets are transmitted in increasing order of the finish tags. Ties are broken arbitrarily.

To emulate SCFQ in which the frame with the smallest finish tag is transmitted first, each transmitted frame in DFS is stamped with a finish tag, based on which the $\mathrm{BI}$ for each competing mobile station is then chosen. The backoff interval $B I_{i}$ for each mobile station $i$ is expressed as

$$
B I_{i}=\left\lfloor\left\lfloor\text { Scaling_Factor } \times \frac{L_{i}}{\phi_{i}}\right\rfloor \times \rho\right\rfloor,
$$

where $L_{i}$ is the head of line frame size and $\rho$ is a random variable uniformly distributed in $[0.9,1.1]$. In (2), the ratio between $L_{i}$ and $\phi_{i}$ is from the finish tag, and the scaling factor allows the choice of an appropriate scale for the virtual time in DFS.

\section{Proportional Fairness Service}

To implement proportional fairness service, each AC is assigned a positive real number $\phi[A C]$ indicating the weight of sharing the channel capacity. The calculation of BI (Backoff-Interval) of the head-of-line packet is based on (2) in DFS. Note that DFS may need a backoff interval mapping scheme to improve the throughout performance as the duration of the backoff interval is inversely proportional to the weight of a station. Any increasing function can be used as the mapping scheme of DFS, and the following square-root mapping scheme proposed in [7] is used in DS-EDCA.

$$
B I_{i}=\psi(\Delta)=\left\{\begin{array}{lc}
\Delta & \text { if } \Delta<\text { Threshold } \\
\lfloor\sqrt{\text { Threshold } \times \Delta}\rfloor & \text { otherwise }
\end{array}\right.
$$

where $\Delta$ denotes the backoff interval obtained in (2) and Threshold is a constant parameter.

\section{Strict Priority Service}

To provide strict priority, higher priority frames must be transmitted before the lower-priority ones. In other words, the AIFS values of the lower priority must be larger than that of the higher priority plus its maximum contention window, i.e.,

$$
\operatorname{AIFS}[j] \geq \operatorname{AIFS}[i]+C W_{\max [i]} \text { if } \mathrm{j}>\mathrm{i}
$$

To sum up, in DS-EDCA, each AC is associated with a backoff entity and an EDCA parameter set, i.e., 
AIFS[AC], $C W \min [\mathrm{AC}]$, and $C W \max [\mathrm{AC}]$, but the value of the AIFS[AC] is now determined by (4). The backoff intervals of strict priority classes are the same as in EDCA and that of proportional classes are calculated by (2).

\section{HIERARCHICAL LINK SHARING IN WLANS}

In 802.11e WLANs, the backoff entities of all mobile stations and the AP contend for the wireless bandwidth in the same way. Since the downlink traffic (from AP to mobiles) to different mobiles must pass through the AP, the downlink traffic may be several times the amount of uplink traffic (from mobiles to AP) sent by each mobile station. As a result, the downlink traffic will be dropped in the AP when the uplink traffic is heavy due to the input rate being larger than the service rate. For example, if there are four pairs of VoIP sessions between mobiles stations, the aggregated downlink VoIP traffic is eight times of the traffic in the uplink direction. This implies that the throughput of the downlink VoIP traffic must be eight times of that in the uplink direction in order for the demanded QoS of VoIP to be ensured. This problem has been ignored in all existing work. In this section, we propose a hierarchical link sharing architecture for 802.11e WLANs, based on which the proposed DS-EDCA mechanism can provide the desired service for different traffic.

Fig. 1 shows the link sharing hierarchy of WLANs with respect to the uplink and downlink traffic. The AP contends with all mobile stations for the downlink bandwidth. Then, it may distribute the link resource to all mobile stations equally, or according to their pre-assigned weights if a weighted-fair discipline is adopted for the downlink traffic. Fig. 2 shows the system architecture of the 802.11e AP with queuing discipline. Before entering the backoff entity of each AC in the AP, the packets to each mobile station are scheduled based on the respective queuing discipline. Then, the backoff entities of AP contend for the downlink bandwidth while those of all mobile stations contend for the uplink bandwidth. Therefore, AP should use different EDCA parameter sets from those of mobile stations if we want to provide differentiated service among downlink and uplink traffic. Moreover, if there is some data traffic such as FTP or Web browsing flows in the system, the link bandwidth in both directions should be assigned to real-time traffic (say, VoIP) first so as to meet the more stringent delay and delay jitter requirements. Suppose each VoIP session needs a rate of $V$ bps to meet the desired QoS and there are four VoIP pairs in the system. Let AC0 be assigned to the VoIP traffic, and AC2 for the data traffic, respectively. Among the high priority classes (i.e., AC0), the backoff entity of AP should share $8 \mathrm{~V}$ bps and that of each mobile station, $V$ bps. The remaining bandwidth is then shared by the uplink and downlink data traffic. Suppose there are $n$ uplink data flows, and the weights of uplink and downlink data traffic are maintained in the ratio of $j: k$. The weights of the backoff entities of low priority ACs (AC2) for AP and each mobile station should be set as $h: i$, where $h: n * i=k: j$, i.e.,

$$
h: i=\frac{n * k}{j} \text {. }
$$

The bandwidth sharing hierarchy is illustrated in Fig. 3, where $C$ denotes the link capacity.

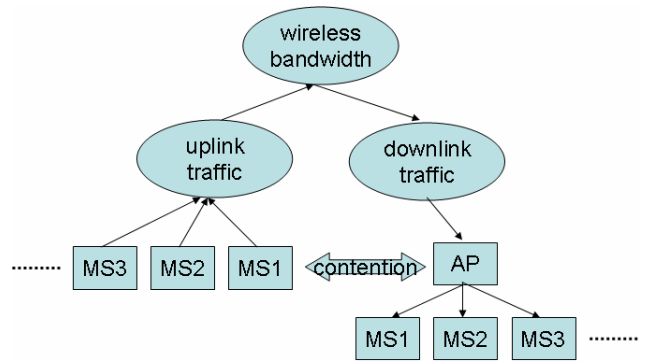

Figure 1. The hierarchical link sharing architecture in WLANs

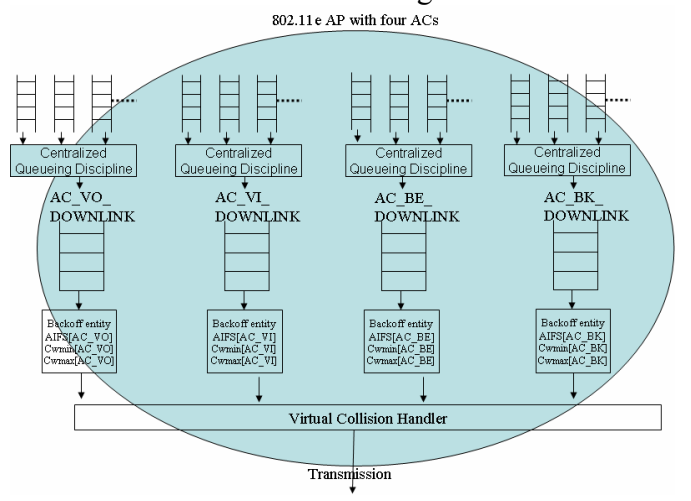

Figure 2. System design for the 802.11e Access Point

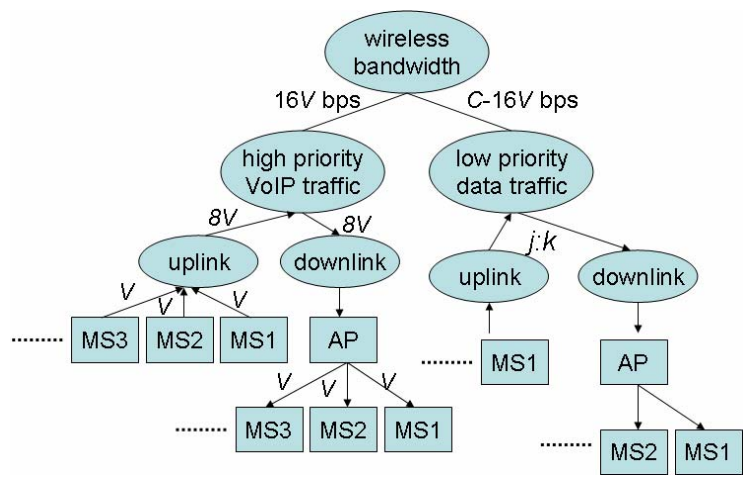

Figure 3. An example hierarchical link sharing architecture

\section{PERFORMANCE EVALUATION}

In this section, the performance of the 802.11e EDCA and DS-EDCA is evaluated with the ns-2 EDCA implementation created by Chesson and Singla [13]. The wireless link rate is $11 \mathrm{Mbps}$ and each data flow is 
associated with one AC and generates CBR UDP traffic at a rate of $8 \mathrm{Mbps}$ to the AP. The packets, including the IP header, of all ACs are fixed at 1520 bytes. The values of the EDCA parameter sets for the four ACs are listed in Table 1.

Table 1. Values of EDCA parameter sets used in the simulations

\begin{tabular}{|l|c|c|c|c|}
\hline & AC0 & AC1 & AC2 & AC3 \\
\hline AIFSN & 2 & 2 & 3 & 7 \\
\hline CWmin & 3 & 7 & 15 & 15 \\
\hline CWmax & 7 & 15 & 1023 & 1023 \\
\hline
\end{tabular}

We first demonstrate the effect of strict priority implemented in our mechanisms for delay-sensitive traffic as compared to the original EDCA. In this simulation, only $\mathrm{AC} 0$ and $\mathrm{AC} 1$ flows are active. Fig. 4 plots the average delay and delay variation of $\mathrm{AC} 0$ as a function of the number of $\mathrm{ACl}$ flows. It shows that the original EDCA is very sensitive to the number of active low priority flows, while in DS-EDCA, the average delays and delay variations are invariant to the traffic load of active low priority. This proves that the proposed mechanism is suitable to provide service for delay sensitive traffic such as VoIP over WLANs.

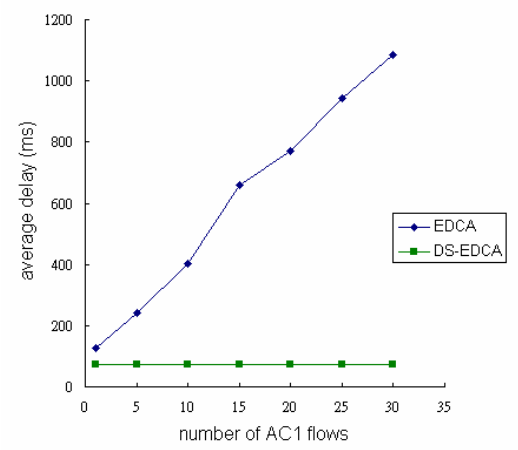

(a) Average delay of AC0

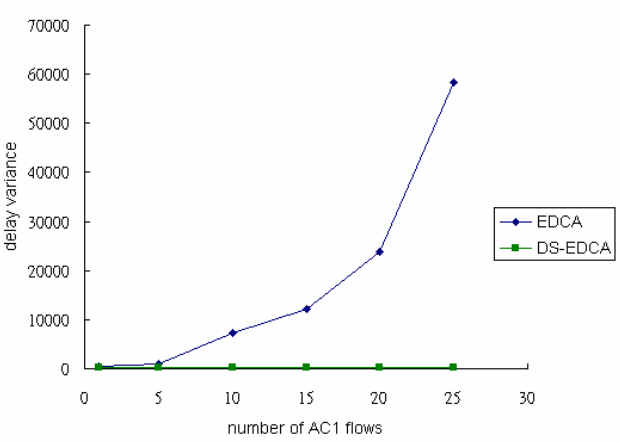

(b) Delay variance of AC0

Figure 4 . The delay and inter-arrival time of flows

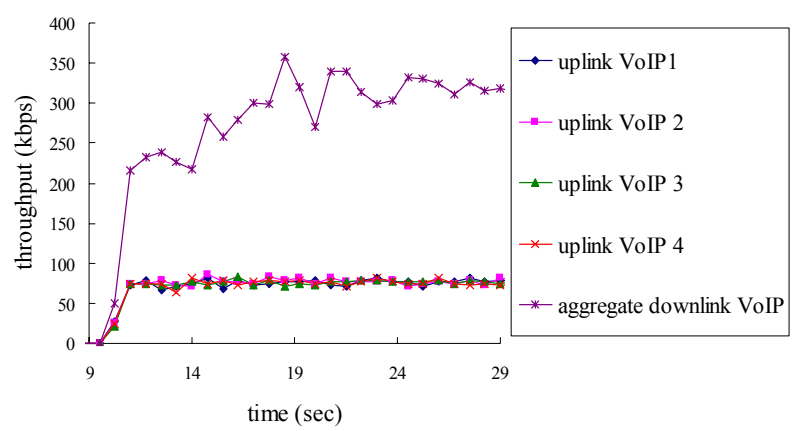

(a) The throughputs of VoIP flows in EDCA

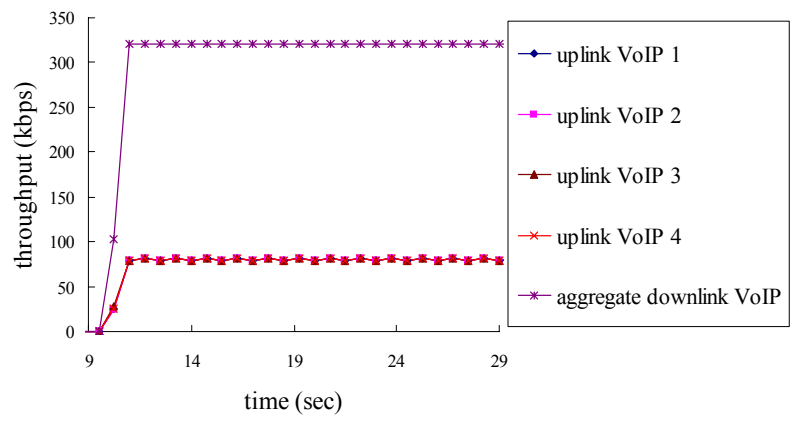

(b) The throughputs of VoIP flows in DS-EDCA

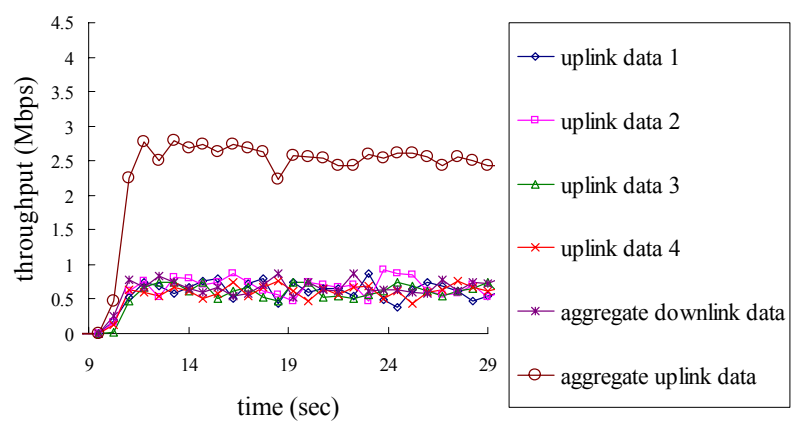

(c) The throughputs of data flows in EDCA

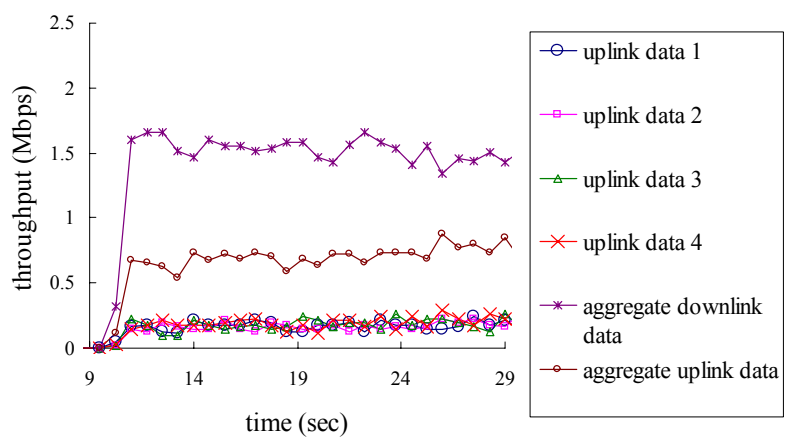

(d) The throughputs of data flows in DS-EDCA

Figure 5. The throughputs of EDCA and DS-EDCA

We then demonstrate the strength of the hierarchical link sharing architecture in 802.11e WLANs. In this simulation, we consider two pairs of G.711 VoIP calls (four uplink and four downlink VoIP flows); four uplink 
and four downlink CBR UDP data flows, each operating at $4 \mathrm{Mbps}$. The objective is to guarantee QoS for VoIP sessions and ensure the aggregate throughputs of uplink and downlink data traffic be in the ratio of $1: 2$. We configure the VoIP traffic and data traffic as AC0 and $\mathrm{AC} 2$, respectively, for the original 802.11e EDCA. In this simulation, only AC0 and AC2 flows are active. For the proposed hierarchical DS-EDCA, the downlink and uplink VoIP flows are configured as AC0, and the weights of backoff entities for AP and all other mobile stations are set to be $4: 1$. The downlink and uplink data flows are $\mathrm{AC} 2$, and their weights between backoff entities of AP and other mobile stations are set to 8:1 according to (5). The AC0 have strict priority over AC2.

Fig. 5 compares the throughput performance of the original EDCA and DS-EDCA with different traffic types. Figs. 5(a) and 5(b) plot the throughputs of VoIP flows for the original EDCA and DS-EDCA, respectively. DSEDCA has a similar throughput variance of uplink VoIP flows as that in the original EDCA, but has a relatively stable and higher throughput for aggregate downlink VoIP traffic because the AP in the original EDCA does not have priority over mobile stations in channel contention. Thus, DS-EDCA can guarantee the demanded QoS for both uplink and downlink VoIP flows. Figs. 5(c) and 5(d) plot the aggregate throughputs of data flows for EDCA and DS-EDCA, respectively. Fig. 5(c) shows that the aggregate throughput of downlink data flows from the AP in EDCA is equal to that of each individual uplink data flow, which may lead to serious packet loss when traffic load is heavy. On the other hand, as shown in Fig. 5(d), the aggregate throughput of downlink data flows is twice that of the aggregate uplink data flows in DS-EDCA, which fits the planned resource management policy (i.e., the ratio of uplink to downlink is 1:2). Besides, each uplink data flow shares an equal amount of bandwidth, and thus the uplink resource is shared fairly. Therefore, the proposed service model can control the bandwidth sharing among downlink and uplink flows according to link sharing policy through the priority and weight assignments implemented in DS-EDCA.

\section{CONCLUSION}

In this paper, we have proposed a service mechanism called Differentiated Service -- Enhanced Distributed Channel Access (DS-EDCA) for IEEE 802.11e WLANs. With DS-EDCA, strict priority and weighted fair service among ACs are provided such that we can control the delay and delay variation of the QoS traffic and ensure proportional bandwidth sharing among the best-effort traffic. The EDCA parameter set of lower priority traffic is carefully designed and the backoff intervals of ACs are determined according to the distributed scheduling discipline DFS. Furthermore, a hierarchical link sharing model for IEEE 802.11e WLANs is proposed, which proves very useful in downlink traffic scheduling. We have also conducted simulations based on ns-2 to compare the performance of IEEE 802.11e EDCA and DS-EDCA in terms of strict priority, proportional service, fairness, and aggregate throughput of each AC. The simulation results show that with DS-EDCA, the ACs can share the channel capacity strictly according to their priorities, assigned weights. Most importantly, DS-EDCA is easily implemented and is compatible with the IEEE 802.11 Standard.

\section{ACKNOWLEDGEMENT}

This work was supported partly by National Science Council under a Center Excellence Grant NSC93-2752-E002-006-PAE, and in part by the National Science Council, Taiwan, under grant number NSC94-2218-E002-059.

\section{REFERENCES}

[1] IEEE WG, Draft Supplement to Standard for Wireless LAN Medium Access Control (MAC) and Physical Layer (PHY) Specifications: Medium Access Control (MAC), Enhancements for Quality of Service (QoS), IEEE 802.11e Draft 4.1, Feb. 2003.

[2] I. Aad and C. Castelluccia, "Differentiation Mechanisms for IEEE," IEEE INFOCOM 2001.

[3] J. Deng and R.S. Chang, "A Priority Scheme for IEEE 802.11 DCF Access Method," IEICE Trans. Commun., Vol. E82-B, no. 1, 1999, pp. 96-102

[4] V. Kanodia, C. Li, A. Sabharwal, B. Sadeghi and E. Knightly, "Distributed Multi-Hop Scheduling and Medium Access with Delay and Throughput Constraints," $A C M$ Mobicom 2001

[5]S. Golestani, “A Self-Clocked Fair Queueing Scheme for Broadband Applications." IEEE INFOCOM'94, page 636646, Toronto, CA, Jane 1994

[6] S. Lu, T. Nandagopal, and V. Bharghavan, "A Wireless Fair Service Algorithm for Packet Cellular Networks," $A C M$ Mobicom 1998.

[7] N. H. Vaidya, P. Bahl, and S. Gupta, "Distributed Fair Scheduling in Wireless LAN,” ACM Mobicom 2000.

[8] D. Qiao and K. G. Shin, “Achieving Efficient Channel Utilization and Weighted Fairness for Data Communications in IEEE 802.11 WALN under the DCF," ACM IWQoS 2002.

[9] W. Pattara-Atikom, S. Banerjee, and P. Krishnamurthy, "Starvation Prevention and Quality of Service in Wireless LANs." IEEE WPMC 2002.

[10] IEEE 802.11, Wireless LAN Medium Access Control (MAC) and Physical Layer (PHY) specifications, Aug. 1999.

[11] M. Malli, Q. Ni, T. Turletti and C. Barakat, "Adaptive Fair Channel Allocation for QoS Enhancement in IEEE 802.11 Wireless LANs.” IEEE ICC 2004.

[12] M. Shreedhar and G. Varghese, "Efficient Fair Queuing Using Deficit Round-robin," IEEE/ACM Trans. Net., vol. 4, no. 3 , 1996, pp. 375-85.

[13] ns-2, available at http://www.isi.edu/nsnam/ns/ 